\title{
Relación entre desarrollo cognitivo y contextos de interacción familiar de niños que viven en sectores urbanos pobres"
}

Relationship between cognitive development and family interaction contexts of children living in poor urban sectors

Recibido: mayo 13 de 2010 | Revisado: noviembre 14 de 2010 | Aceptado: mayo 2 de 2011

\author{
Mariela Orozco-Hormaza ** \\ HERNÁN SÁNCHEZ-RÍOs*** \\ Universidad del Valle, Cali, Colombia \\ Elda Cerchiaro-Ceballos ***** \\ Universidad del Magdalena, Santa Marta, Colombia
}

Para citar este artículo. Orozco-Hormaza, M., Sánchez-Ríos, H. \& Cerchiaro-Ceballos, E. (2012). Relación entre desarrollo cognitivo y contextos de interacción familiar de niños que viven en sectores urbanos pobres. Universitas Psychologica, 11(2), 427440 .

Este artículo presenta los resultados de la investigación titulada "El desarrollo infantil en entornos urbanos pobres y contextos de interacción", COLCIENCIAS, 2007 (1106-331-18930), presentada por el Grupo Desarrollo Psicológico en Contextos del Centro de Investigaciones en Psicología, Cognición y Cultura de la Universidad del Valle y por el Grupo Cognición y Educación de la Universidad del Magdalena.

* Grupo de Desarrollo Psicológico en Contextos, Universidad del Valle, Cali, Colombia. Cl. 13B 85-24, Apto. 304. Cali, Colombia. E-mail: orozcohormaza@gmail.com

**** Grupo de Desarrollo Psicológico en Contextos, del Centro de Investigaciones en Psicología, Cognición y Cultura, de la Universidad del Valle, Cali, Colombia.E-mail: hesanche@univalle.edu.co

***** Grupo Cognición y Educación. Programa de Psicología. Universidad del Magdalena, Santa Marta, Colombia. Carrera 32 № 22-08 Sector San Pedro Alejandrino. E-mail: elda.cerchiaro@gmail.com

\section{RESUMEN}

Este artículo relaciona el desarrollo cognitivo de 405 niños de 3, 4 y 5 años, que viven en sectores urbanos pobres de Cali y Santa Marta (Colombia), con dimensiones que describen sus contextos de interacción familiar. Como indicador de desarrollo se utilizaron las estrategias de clasificación que utilizan individualmente los niños en la resolución de un problema, y como descriptores de los contextos de interacción, el nivel de pobreza de la familia y las prácticas y expectativas de los padres. Los resultados muestran que el desarrollo cognitivo alto no se relaciona con el nivel de pobreza de la familia, como dimensión aislada, sino con niveles altos de prácticas de formación, entretenimiento, protección y regulación del comportamiento. Palabras clave autores

Desarrollo cognitivo, contextos de interacción familiar, clasificación, prácticas de cuidado.

Palabras clave descriptores

Prácticas de formación, expectativas de los padres, cognición, psicología social.

\section{A B S T R A C T}

This article relates the cognitive development of 405 children aged 3, 4 and 5, living in urban poor in Cali and Santa Marta (Colombia), with dimensions that describe their contexts of family interaction. As an indicator of development were used strategies classification used in solving a problem, and as descriptors of contexts of interaction, the level of poverty, practices and parental expectations. The results show that cognitive development is not related to high poverty levels, as isolated dimension, but with high levels of practices training, entertainment, protection and regulation of behavior. Key words authors

Cognitive development, family interaction contexts, classification, care practices. Key words plus

Training practices, parental expectations, cognition, social psychology. 


\section{Introducción}

Este artículo presenta datos recientes de un estudio realizado con niños que viven en sectores urbanos en ciudades colombianas. La investigación se llevó a cabo con el fin de explorar las relaciones posibles entre el desarrollo cognitivo de estos niños, indicado a partir del uso de la clasificación en una situación de resolución de problemas, y las dimensiones y variables que definen el contexto de interacción familiar en el que ellos crecen ${ }^{1}$.

\section{Aproximación al concepto de desarrollo cognitivo}

Entendemos el desarrollo como un proceso integral de construcción y cambio, en el cual los niños participan activamente; proceso que involucra transformaciones en las esferas cognitiva, afectiva y social que afectan a cada niño como un todo y lo definen como persona en un contexto dado (Orozco, Perinat \& Sánchez, 2009). Desde este marco general, el desarrollo cognitivo se define en función de los cambios que se operan en el niño y le permiten construir progresivamente diferentes tipos de capacidades, estructuras y modalidades de funcionamiento para responder a las demandas del contexto. Estos cambios no necesariamente coinciden con una edad determinada y pueden resultar de la interacción dinámica entre las características de los niños y las condiciones de los contextos en los que interactúan (Orozco et al., 2009).

El desarrollo cognitivo describe el cambio en períodos largos de tiempo y se diferencia del cambio cognitivo, que lo describe en períodos cortos, algunas veces en tiempo real. Por lo tanto, hay una distancia con investigaciones que estudian cómo se produce el cambio cognitivo (Siegler, 2000) o los mecanismos subyacentes (Puche, 2003).

Respecto a los determinantes del desarrollo (Puche, 2008) en esta investigación se adopta una perspectiva que se ha denominado desarrollo cognitivo en contextos (Orozco et al., 2009). Esta posición

1 Conviene precisar que las expresiones niños o niño se utilizan para designar los dos géneros. sugiere que las prácticas concretas que los niños asumen y realizan en sus contextos de interacción y los instrumentos de control que allí encuentran, generan experiencias que contribuyen al cambio y a la construcción permanente de conocimiento, a partir de mecanismos de autorregulación propios (Pascual-Leone \& Johnson, 1991), que especifican los contenidos de sus procesos de construcción de conocimiento. Estas prácticas obedecen a demandas de los contextos en los que los niños interactúan. En otras palabras, el contexto provee las prácticas cotidianas de las cuales los niños derivan experiencias que definen los contenidos de sus procesos cognitivos y les permiten construir categorías acerca del mundo físico y social con el cual interactúan.

\section{La clasificación como indicador de desarrollo cognitivo}

Desde temprana edad, los niños son capaces de construir categorías con los objetos, clasificarlos de acuerdo con sus semejanzas y ordenarlos en función de sus diferencias (Karmiloff-Smith, 1994). Esto supone agruparlos con arreglo a determinadas condiciones o cualidades. De esta manera, los niños descubren progresivamente los principios de la llamada lógica a partir del uso de la clasificación (Puche, 2000).

Esta operación cognitiva aparece tempranamente en los niños, primero bajo la forma de colecciones y después de clases propiamente dichas (Piaget \& Inhelder, 1976). Una clase se define a partir de las relaciones entre los elementos que la conforman y la clase de la que hacen parte, bajo el criterio de la pertenencia inclusiva. Estos autores definen dos criterios centrales que caracterizan los sistemas de clases: la comprehensión y la extensión. Es decir, solo se puede hablar de clases cuando el niño es capaz de definirlas en comprehensión, a partir de las cualidades comunes a sus miembros y manipularlas en extensión, teniendo en cuenta las relaciones de inclusión y pertenencia inclusiva.

Estos autores proponen dos tipos de clasificaciones: clasificación aditiva y clasificación multiplicativa. En la clasificación aditiva el niño construye un 
sistema de clases, establece relaciones de diferencia, relaciones de semejanza entre los elementos de las clases, relaciones de inclusión que definen la pertenencia inclusiva y maneja relaciones de complementariedad en las que identifica subclases complementarias que conforman una clase $(\mathrm{B}=\mathrm{A}+\mathrm{no} \mathrm{A})$. Además de los criterios de la clasificación aditiva, para la clasificación multiplicativa se utilizan otros criterios: 1) "todos los elementos de B1 pertenecen también a B2 y recíprocamente" ... ; 2) "todos los elementos de A1 pertenecen a A2 o a A'2," ... "pero no a ambos a la vez en virtud del criterio de disyunción"; 3) "las subclases A1 y A'1 solamente comprenden elementos que pertenecen también a A2 o a A'2" ... "y las subclases A2 y A'2 solo comprenden elementos que pertenecen también a $\mathrm{A} 1 \mathrm{o}$ a A'1" y 4) "cada asociación elemental A1 A2 o A'1 A'2 constituye una y solo una clase multiplicativa" (Piaget \& Inhelder, 1976, p. 169).

Para investigar el desarrollo de la clasificación se adopta la metodología de las Situaciones de Resolución de Problemas (SRP), que permite explorar los procesos mentales que los niños despliegan al resolverlos, y seguir la trayectoria de su pensamiento a través de la secuencia de acciones y verbalizaciones que ponen al descubierto las estrategias y esquemas de acción subyacentes a su desempeño (Thornton, 1998). El enfoque de la SRP se centra en la actividad del niño y no en respuestas verbales o acciones puntuales. Su estructura medio-fin desvirtúa el acierto y error como único recurso de resolución.

\section{Los contextos de interacción familiar}

En su modelo ecológico, Bronfenbrenner (1979) distingue cuatro sistemas, relacionados entre sí, que inciden en el desarrollo humano. En primera instancia, describe los microsistemas como patrones de actividades, roles y relaciones interpersonales en los que la persona participa, en un entorno determinado. La familia, sus prácticas, sus integrantes y relaciones ejemplifican un microsistema. Las interrelaciones entre dos o más entornos en los que las personas interactúan constituyen el meso-sistema; en este caso, las relaciones entre la familia y el hogar comunitario ilustran esta dimensión. El exo-sistema se refiere a uno o más entornos que no incluyen a la persona en desarrollo como participante activo, pero en los cuales ocurren hechos que afectan su entorno directo; de esta manera, las jornadas laborales de los padres y las opciones de programas de protección y cuidado para los niños mientras sus padres trabajan, son ejemplos de este sistema. Por último, el macro-sistema está constituido por el sistema de creencias o ideologías que sustentan los anteriores.

De acuerdo con la edad de los niños participantes en este estudio -3 a 5 años- y sus condiciones de vida, el meso-sistema enmarca las actividades y relaciones en el contexto de interacción familiar y se pregunta por las características de los componentes de cada microsistema que potencian y favorecen su desarrollo cognitivo.

Desde esta perspectiva, la familia se define en función de un marco de actividad molar (Bronfenbrenner, 1979), es decir, acciones o actividades específicas que poseen un momento propio y tienen significado o intención para los que participan en ellas, que en esta investigación se denomina prácticas. Bronfenbrener las concibe como acciones, es decir, como comportamientos intencionados y regulados que los adultos encargados de los niños efectivamente asumen y que favorecen su aprendizaje, crecimiento y desarrollo.

Rodrigo (1994) entiende los contextos como escenarios socioculturales en los cuales los niños construyen conocimiento. Para esta autora, los contextos constan de un entorno físico, con actores que interactúan en el curso de actividades específicas, movidos por propósitos y metas que tratan de comunicar y negociar unos con otros. Los contextos varían en función de "los entornos físicos, los actores que intervienen, sus vínculos interpersonales, sus intenciones y metas, el tipo de actividades socioculturales que realizan y los procesos de negociación que llevan a cabo" (p. 32).

El niño, sus padres, los hermanos que viven en la misma casa u otros familiares e incluso personas ajenas a la familia pero que comparten la vivienda, pueden ser considerados como actores en la vida de los niños. Sin embargo, esta denominación resulta insuficiente, si no se establece el tipo de relación 
que tienen con ellos y las prácticas que asumen en el marco de la vida familiar.

Myers (2003) establece como indicadores del contexto, que permiten caracterizar condiciones que pueden afectar directamente el desarrollo de los niños, las siguientes dimensiones: "estructura familiar, características de los padres y/o del adulto que cuida del niño, estado de empleo de los padres, condiciones de la vivienda, condiciones sociales y económicas generales de la familia y su acceso a servicios" (p. 166). De otro lado, García-Coll (1990) propone las creencias culturales y prácticas de crianza, las condiciones de salud y prácticas de cuidado de la salud, factores biológicos y la procedencia étnica como condiciones que influyen en el desarrollo de infantes.

Las prácticas de crianza y las expectativas de los padres frente al futuro de sus hijos constituyen condiciones que pueden incidir directamente en el desarrollo de los niños (Orozco et al., 2009). En esta perspectiva, Myers (2003) define como indicadores de prácticas de crianza las relativas a la salud, alimentación e interacciones con los niños, su socialización y las modalidades de disciplina o abuso que se dan en la familia. Orozco et al. sintetizan estas propuestas y diferencian tres tipos de prácticas que definen las funciones que los adultos cumplen con los niños y que se adoptan para este estudio: prácticas de entretenimiento, de formación y de protección y cuidado.

En relación con las expectativas, LeVine (1977) señala que todos los pueblos del mundo comparten tres tipos de metas o expectativas para sus hijos: 1) de supervivencia, cómo lograr la salud física del niño, 2) formación para el oficio, aprendizaje a través de instrucciones o actividades supervisadas de un oficio y 3) de formación moral, valores, normas y principios, tipos de creencias que lo rijan y lo formen.

La caracterización de la organización familiar, las condiciones socioeconómicas de las familias, las prácticas de entretenimiento, formación, protección y cuidado y de regulación del comportamiento que los padres implementan con sus hijos y las expectativas sobre su futuro, permitieron a la presente investigación definir el contexto de interacción fa- miliar y crear el marco que define las dimensiones y variables constituyentes del contexto familiar.

\section{Desarrollo cognitivo y contextos de interacción familiar}

La investigación que estudia la relación entre desarrollo cognitivo y contextos familiares no es reciente (Bronfenbrenner, 1979; Daza, 1999; Gauvain, 1995; Gónzalez, 1999; Greenfield \& Lave, 1982; Myers, 2003; Rodrigo, 1994; Rodríguez, 2006; Rogoff, 1993; Shonkoff \& Phillips, 2000; Valsiner, 2007), pero ha tomado un progresivo interés en los estudios de desarrollo de los niños que crecen en contextos urbanos pobres (Amar, 1994; Bruner, 1975; GarcíaColl, 1990; Korenman, Millar \& Sjaastad, 1994; Marquez dos Santos, Neves \& Lima, 2010; Mcloyd, 1990, 1998; Orozco et al., 2009; Ross \& Roberts, 1999; Tudge, 2009) y en comunidades específicas (Chavajay, 2006; Correa-Chávez \& Rogoff, 2009; Greenfield, Maynard \& Childs, 2000; Rogoff, Correa-Chávez \& Silva, 2011; Rogoff, Mistry, Göncü \& Mosier, 1993; Rogoff et al., 2007).

Las investigaciones muestran las siguientes tendencias: 1) el impacto del nivel socioeconómico de las familias pobres en el desarrollo de los niños (Korenman et al., 1994; Mcloyd, 1990); 2) la descripción de la relación entre desarrollo cognitivo y condiciones del contexto familiar (Orozco et al., 2009) y 3) las extraordinarias capacidades cognitivas y sociales de los niños pequeños al participar en prácticas propias de sus comunidades de origen (Chavajay, 2006; Correa-Chávez \& Rogoff, 2009; Greenfield et al., 2000; Orozco, Ochoa \& Sánchez, 2001; Rogoff et al., 1993; Rogoff et al., 2007). En el interés por explorar estas relaciones, en el presente estudio se asume como hipótesis, por un lado, que existe relación entre el desarrollo cognitivo de los niños y sus contextos de interacción y, por otro lado, que los tipos de prácticas y las actividades que los padres y cuidadores realizan en su interacción con los niños influyen en su desarrollo.

Las siguientes preguntas guiaron la investigación: ¿Cuál es el nivel de desarrollo cognitivo en que se encuentran los niños?, ¿Qué caracteriza sus contextos de interacción familiar?, ¿Qué relación 
existe entre los contextos de interacción y el nivel de desarrollo cognitivo encontrado en los niños?

\section{Método}

Para describir el desarrollo cognitivo de los niños y las posibles relaciones con los contextos de interacción familiar, se utilizó un diseño transversal de carácter exploratorio.

\section{Participantes}

En el estudio participaron 405 niños entre 3 y 5 años, de ambos sexos (204 en Cali y 201 en Santa Marta), quienes resolvieron un problema que exigía el uso de la clasificación. La muestra fue intencional y estuvo constituida de la siguiente manera: 134 niños de 3 años (edad promedio 41 meses, $D E=3.8) ; 136$ niños de 4 años (edad promedio 53 meses, $D E=3.4$ ); 135 niños de 5 años (edad promedio 64 meses, $D E=3.7$ ). Los niños participantes asisten a Hogares Comunitarios (HC) del Instituto Colombiano de Bienestar Familiar: $40 \mathrm{HC}$ en Cali y 56 en Santa Marta ${ }^{2}$ y sus familias pertenecen a los niveles 1 y 2 del SISBEN ${ }^{3}$.

\section{Instrumento para describir el desarrollo cognitivo}

Para estudiar la clasificación se utilizó una SRP denominada "Las caritas" (Puche, 2003). El problema exige que el niño encuentre cuál es la carita que el entrevistador(a) ha escogido -sin que el niño se dé cuenta- entre 16 caritas dispuestas en un tablero. Inicialmente, el(la) entrevistador(a) familiariza al niño con el problema; para esto le pide que escoja una carita y le formula preguntas cuyas respuestas le permiten descubrirla. Después le dice: "Bien, ahora debes descubrir cuál es la carita que yo escogí. Para encontrarla me puedes hacer las preguntas que

2 El Hogar Comunitario es una institución financiada por el Estado colombiano y atiende niños con familias que viven en sectores urbanos pobres.

3 Sistema de Identificación de Potenciales Beneficiarios de Programas Sociales. quieras sobre las caritas que tienes en el tablero" (Figura 1).

La clasificación multiplicativa define la estructura del problema (Pascual-Leone \& Johnson, 2004). Esta operación se establece cuando se presenta una intersección de dos o más dimensiones de clases aditivas. Dadas dos clases $(\mathrm{B} 1=\mathrm{A} 1+$ $\mathrm{A}^{\prime} 1$ y $\left.\mathrm{B} 2=\mathrm{A}_{2}+\mathrm{A}^{\prime} 2\right)$, los elementos se clasifican al mismo tiempo según los criterios de la clase B1 (género) y de la clase B2 (color de camiseta), lo que da origen a cuatro subclases distintas. La estructura de este problema exige intersectar cuatro clases, definidas por los atributos de las caritas: género, color de camiseta, gesto y color de la piel. Por cada atributo se consideran dos rasgos, así: género $\mathrm{A}$ : $\mathrm{A} 1=$ niños $\mathrm{A}^{\prime} 1=$ niñas; gesto $\mathrm{B}: \mathrm{B} 1=$ sonriente $\mathrm{B}^{\prime} 1$ = triste; color $\mathrm{C}: \mathrm{C} 1$ = blanco $\mathrm{C}^{\prime} 1$ = negro; color de camiseta D: D1 = azul D'1 = roja.

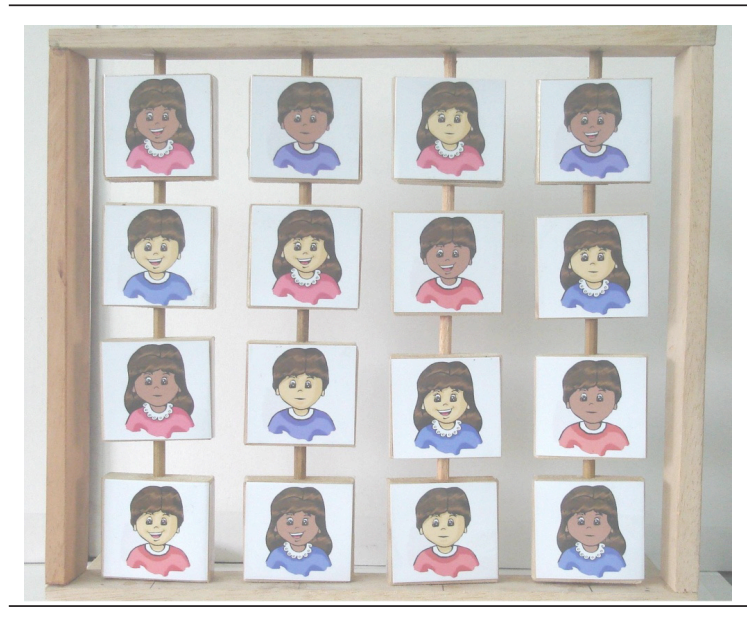

Figura 1. Tablero de las caritas.

Fuente: elaboración propia.

Para identificar la carita escogida por el(la) entrevistador(a), el niño debe establecer: 1) Relaciones de diferencia, es decir, identificar los atributos y rasgos que caracterizan a los elementos de una clase y diferenciarlos de los de la otra; 2) Relaciones de semejanza entre miembros de una clase, así: todas las niñas (A) pertenecen al mismo género; 3) Relaciones de inclusión y de pertenencia inclusiva: en la clase de las "caritas" hay algunos que son niños y otros que son niñas; 4) Relaciones de complementariedad, esto es, identificar elementos que forman parte de 
una clase; por ejemplo, la clase de "las caritas" está conformada por niños y no niños (las niñas) y 5) Interacciones entre los rasgos de las cuatro clases.

\section{Instrumento para describir los contextos}

El instrumento utilizado para describir los contextos familiares de los niños del estudio fue la Encuesta del Adulto Cuidador, adaptada de estudios previos (Orozco et al., 2009; Panel Study of Income Dynamics [PSID], 1997). La encuesta utiliza tres tipos de preguntas: aquellas que exigen respuestas dicotómicas: sí o no, las de opciones excluyentes y las de respuesta múltiple. La encuesta se aplicó a 405 adultos cuidadores de los niños, de los cuales el $76 \%$ fue el padre o la madre y el $24 \%$, algún familiar que vive en la misma casa que habita el niño.

\section{Modalidades de análisis}

Para obtener un indicador del desarrollo cognitivo de cada niño al resolver el problema, se utilizaron tres niveles jerárquicos de análisis. El primer nivel identifica su desempeño o logro: si encuentra la carita escogida por el(la) entrevistador(a), (1) o no la encuentra (0). El segundo nivel permite inferir la estrategia que utiliza para identificar la carita escogida; en el tercero, las demandas cognitivas del problema sirven de marco para inferir los esquemas de acción que guían la actividad del niño. Estos esquemas se utilizan como criterio para establecer el cambio de una estrategia menos elaborada a otra más elaborada. La presencia de un nuevo esquema de acción, que involucre elementos más complejos de la clasificación, permite diferenciar un nuevo tipo de estrategia de resolución. La Tabla 1 presenta la descripción de las ocho estrategias identificadas y los esquemas de acción que las fundamentan.

A cada tipo de estrategias se le asignó un puntaje según el orden de complejidad, definido en función del correspondiente tipo de esquemas de acción subyacente. La sumatoria del puntaje de la estrategia y del desempeño de cada niño permite establecer la puntuación que se asigna al proceso de solución, la cual se incrementa en cinco puntos de acuerdo con los cambios cualitativos en los tipos de esquemas de acción utilizados.

Para el análisis de correspondencias múltiples, se crearon niveles tanto para el tipo de proceso que los niños utilizan para resolver los problemas que dan cuenta de su desarrollo cognitivo -el problema de clasificación- como para cada una de las dimensiones que describen los contextos de interacción familiar. Para el desarrollo de la clasificación se identificaron cuatro niveles, en función de la complejidad de los esquemas: caritas bajo (CARB: puntajes 1 , $2,3,4)$, caritas medio bajo (CARMB: puntajes 10, 11, 12, 13), caritas medio alto (CARMA: puntajes 17, 18, 19, 20), caritas alto (CARA: puntajes 25, 26, 27, 28). Los niveles de las dimensiones del contexto se basan en la distribución de la población en los cuartiles y en la distribución normal (en aquellos casos en que la dimensión se aproximaba a una distribución normal).

En relación con los contextos de interacción familiar, cada pregunta de la encuesta corresponde a una variable. Para la transformación de las variables se utilizó el siguiente procedimiento: 1) valoración y categorización de las respuestas según los siguientes criterios, convenidos por los investigadores: favorece o no favorece al niño; 2) sumatoria de los valores definidos para cada variable; 3) obtención del porcentaje correspondiente al nivel alcanzado por cada familia en función del puntaje máximo posible para cada dimensión y 4) categorización de la variable en niveles, de acuerdo con los cuartiles obtenidos a partir de la distribución de la población. De manera general, se identificaron entre 3 y 4 niveles. La Tabla 2 describe los niveles asignados a cada dimensión.

Para establecer las relaciones entre desarrollo cognitivo y contexto de interacción se utilizó el método de Análisis de Correspondencias Múltiples, que permite analizar patrones de relaciones de diversas variables, de tipo nominal, en niveles diferenciados. La presentación del Análisis de Correspondencia Múltiple se hace por medio de un gráfico configurado por dos ejes que se cruzan en un punto cero. Las dimensiones que generan aportes altos a la explicación son aquellas que están separadas de los ejes y cercanas entre si. Entre más próxima esté 
TABLA 1

Estrategias de resolución, sus puntajes y esquemas de acción

\begin{tabular}{|c|c|c|}
\hline Estrategia & $\mathrm{P}^{4}$ & Esquemas de Acción \\
\hline Usa deícticos sin orden & 1 & Relación de correspondencia entre la expresión verbal y el señalamiento. \\
\hline $\begin{array}{l}\text { Usa deícticos siguiendo un } \\
\text { orden }\end{array}$ & 2 & $\begin{array}{l}\text { Correspondencia uno a uno entre la expresión verbal y el señalamiento. } \\
\text { Relación de orden. }\end{array}$ \\
\hline $\begin{array}{l}\text { Pregunta por rasgos sin } \\
\text { seguir orden }\end{array}$ & 3 & $\begin{array}{l}\text { Relación de diferencias entre los rasgos de un mismo atributo. } \\
\text { Relación de semejanza entre elementos de una subclase. }\end{array}$ \\
\hline $\begin{array}{l}\text { Pregunta por los rasgos } \\
\text { de un atributo y utiliza } \\
\text { deícticos siguiendo un } \\
\text { orden }\end{array}$ & 4 & $\begin{array}{l}\text { Relación de diferencias entre los rasgos de un mismo atributo. } \\
\text { Relación de semejanza entre elementos de una subclase. } \\
\text { Correspondencia uno a uno entre la expresión verbal y el señalamiento. } \\
\text { Relación de orden. }\end{array}$ \\
\hline $\begin{array}{l}\text { Encadena preguntas } \\
\text { relativas a los rasgos } \\
\text { afirmativos de varios } \\
\text { atributos y señala }\end{array}$ & 5 & $\begin{array}{l}\text { Relación de diferencias entre los rasgos de un mismo atributo. } \\
\text { Relación de semejanza entre elementos de una subclase. } \\
\text { Relación de pertenencia inclusiva. } \\
\text { Intersecciones sucesivas entre, al menos, dos dimensiones o rasgos. }\end{array}$ \\
\hline $\begin{array}{l}\text { Encadena preguntas } \\
\text { simultáneas sobre los rasgos } \\
\text { de los atributos }\end{array}$ & 6 & $\begin{array}{l}\text { Relación de diferencias entre los rasgos de un mismo atributo. } \\
\text { Relación de semejanza entre elementos de una subclase. } \\
\text { Relación de pertenencia inclusiva. } \\
\text { Subclases dentro de una clase. } \\
\text { Intersecciones sucesivas entre más de dos dimensiones o rasgos. }\end{array}$ \\
\hline $\begin{array}{l}\text { Encadena preguntas de dos } \\
\text { atributos, señala o pregunta } \\
\text { por rasgo opuesto }\end{array}$ & 7 & $\begin{array}{l}\text { Relación de diferencias entre los rasgos de un mismo atributo. } \\
\text { Relación de semejanza entre elementos de una subclase. } \\
\text { Relación de pertenencia inclusiva. } \\
\text { Rasgos disyuntos y configura subclases dentro de una clase. } \\
\text { Intersección entre dos o más dimensiones en una operación multiplicativa simple. } \\
\text { Sistema de clases, con base en relaciones de complementariedad. }\end{array}$ \\
\hline $\begin{array}{l}\text { Encadena preguntas } \\
\text { sobre rasgos afirmativos u } \\
\text { opuestos de tres o cuatro } \\
\text { atributos }\end{array}$ & 8 & $\begin{array}{l}\text { Relación de diferencias entre los rasgos de un mismo atributo. } \\
\text { Relación de semejanza entre elementos de una subclase. } \\
\text { Relación de pertenencia inclusiva. } \\
\text { Subclases dentro de una clase. } \\
\text { Intersecciones sucesivas entre más de dos dimensiones en una operación multipli- } \\
\text { cativa compleja. } \\
\text { Sistema de clases, con base en relaciones de complementariedad. }\end{array}$ \\
\hline
\end{tabular}

Nota. $\mathrm{P}^{4}$ : Puntaje.

Fuente: elaboración propia.

una dimensión al eje, es decir, más cercana a cero, su aporte a la explicación es menor.

\section{Resultados}

Los resultados se presentan en tres secciones. La primera incluye los resultados obtenidos al describir el desarrollo cognitivo de los niños al resolver el problema de clasificación. La segunda, los resultados relativos al contexto de interacción familiar y la tercera, los resultados del análisis de correspondencias múltiples entre los niveles de desarrollo alcanzados por los niños en la clasificación y las dimensiones del contexto.

\section{Descripción del desarrollo cognitivo de los niños}

Los niveles de desempeño al resolver el problema, fueron significativamente altos para los tres grupos de edad: $80.6 \%$ para los niños de 3 años, $90.4 \%$ para los de 4 años y $97.8 \%$ los de 5 años.

En cuanto a los puntajes obtenidos en el proceso de solución (Tabla 4), en todos los grupos 
TABLA 2

Variables y niveles alcanzados en cada dimensión del contexto de interacción familiar

\begin{tabular}{ll}
\hline Dimensión & Indicadores y Niveles \\
\hline Nivel socioeconómico & Se identifican tres niveles de pobreza \\
& Alto (NPA): los más pobres \\
& Medio (NPM): pobreza intermedia \\
& Bajo (NPB): los menos pobres \\
Organización Familiar & Se adoptaron dos criterios \\
& Familia monoparental / Nuclear \\
& Con/o sin red de apoyo \\
Prácticas de protec- & Se identifican cuatro niveles de prácticas: \\
ción, entretenimiento, & Prácticas de entretenimiento, formación, protección y cuidado y regulación bajas (P/E/F/ \\
formación y regula- & PC/RC/B) \\
ción del comporta- & Prácticas de entretenimiento, formación, protección y cuidado y regulación medias bajas \\
miento & (P/E/F/PC/RC/MB) \\
& Prácticas de entretenimiento, formación, protección y cuidado y regulación medias altas (P/ \\
& E/F/PC/RC/MA) \\
& Prácticas de entretenimiento, formación, protección y cuidado y regulación altas (P/E/F/ \\
& PC/RC/A) \\
& Se identifican cuatro niveles para las expectativas de los padres: \\
& Expectativas bajas (EXPB) \\
& Expectativas medias bajas (EXPMB) \\
& Expectativas medias altas (EXPMA) \\
Expectativas altas (EXPA)
\end{tabular}

Fuente: elaboración propia

de edad los mayores porcentajes de niños están asignados a los puntajes 2 ( 3 años: $46 \%$; 4 años: $25 \%$; 5 años: $24 \%$ ) y 4 (3 años: $27 \%$; 4 años: $36 \%$ y 5 años: $29 \%$ ), que corresponden al nivel bajo (puntajes 1, 2, 3 y 4). Niños de todas las edades alcanzan los puntajes 9,10 y 12 , correspondientes a los procesos de nivel medio bajo, con un mayor porcentaje de niños de 4 (13\%) y 5 años (14 \%) en el puntaje 10 y de 5 años (13\%) para el puntaje 12. A los 3 años, $7 \%$ de los niños utilizan un proceso de solución de nivel medio alto (puntaje 18), que hace posible encontrar la carita. A los 4 años, el $7 \%$ de los niños utilizan procesos de solución medios altos (puntajes 17 y 19), con los cuales no logran encontrar la carita, mientras que el $5 \%$ y el $3 \%$ llegan a los puntajes 18 y 20 , respectivamente, que permiten encontrarla. En el grupo de 5 años, el $8 \%$ y $5 \%$ de los niños logra utilizar procesos de nivel medio alto (puntajes 18 y 20). En los puntajes que corresponden a los procesos de nivel alto (puntajes $25,26,28$ ), $7 \%$ de niños de 3 y 4 años obtienen una puntuación de
25, con la cual no logran encontrar la carita; a los de 4 y 5 años alcanzan el mismo porcentaje en la puntuación 26 (3.7\%), que hace posible encontrar la carita; solamente los niños de 5 años alcanzan un $3.7 \%$ en el puntaje 28 , que igualmente permite encontrar la carita.

La Figura 2 presenta la distribución de los puntajes para cada grupo de edad. Tal distribución muestra que la mediana es ligeramente superior al puntaje 2 en los grupos de 4 y 5 años y no en el grupo de 3 años. El porcentaje de niños de 4 y 5 años es mayor en los cuartiles 3 y 4 , correspondientes a los puntajes de más alto nivel, que el porcentaje de niños de 3 años; sin embargo, niños de todas las edades, incluidos los de 3 años, alcanzan un puntaje alto, por encima del puntaje máximo en su propia distribución.

\section{Descripción de los contextos de interacción}

En relación con el nivel socioeconómico de las familias se identificaron tres niveles. La mayoría de 
las familias pertenecen al nivel medio de pobreza (57\%), esto es, habitan en una casa o habitación arrendada; las viviendas tienen acceso al menos a tres servicios públicos, están construidas en cemento y ladrillo con piso de cemento, cuentan con una zona común (sala o comedor), el baño dispone de inodoro, ducha, lavamanos y cortina; la cocina tiene muebles y utensilios.

TABLA 3

Distribución de porcentajes de niños en función de puntajes en proceso de solución y edad

\begin{tabular}{lccc}
\hline Puntaje en & \multicolumn{3}{c}{ Edad } \\
\cline { 2 - 4 } Caritas & 3 & 4 & 5 \\
\hline 1 & $10.4 \%$ & $5.9 \%$ & $1.5 \%$ \\
2 & $46.3 \%$ & $25 \%$ & $23.7 \%$ \\
3 & $1.5 \%$ & $0 \%$ & $0.7 \%$ \\
4 & $26.9 \%$ & $36 \%$ & $28.9 \%$ \\
9 & $6.7 \%$ & $1.5 \%$ & $0 \%$ \\
10 & $5.2 \%$ & $13.2 \%$ & $14.1 \%$ \\
12 & $1.5 \%$ & $4.4 \%$ & $10.4 \%$ \\
17 & $0 \%$ & $0.7 \%$ & $0 \%$ \\
18 & $0.7 \%$ & $5.1 \%$ & $8.1 \%$ \\
19 & $0 \%$ & $0.7 \%$ & $0 \%$ \\
20 & $0 \%$ & $2.9 \%$ & $5.2 \%$ \\
25 & $0.7 \%$ & $0.7 \%$ & $0 \%$ \\
26 & $0 \%$ & $3.7 \%$ & $3.7 \%$ \\
28 & $0 \%$ & $0 \%$ & $3.7 \%$ \\
\hline
\end{tabular}

Fuente: elaboración propia.

El $25 \%$ de las familias se ubica en el nivel de mayor pobreza, sus viviendas están construidas en terrenos ilegales o en calidad de tenencia, viven en ranchos arrendados o de invasión, que tienen dos puertas y no tienen ventanas. La vivienda solo posee una habitación que usan para dormir y cocinar, no cuentan con dotación en los baños y en la cocina solamente tienen utensilios para preparar los alimentos. Las paredes de la casa son de madera burda y los pisos de cemento-gravilla. Tienen acceso al servicio de energía eléctrica, cocinan con leña o carbón de leña y obtienen el agua de un pozo con bomba o de un aljibe. Solo el padre recibe un ingreso regular y es quien aporta al sostenimiento del hogar. Los padres tienen dificultades para pagar el arriendo, conseguir dinero para el mercado, pagar los gastos médicos, los medicamentos y comprar ropa. Algunos de ellos vinculan a los niños al Hogar Comunitario para recibir ayuda alimentaría, donde no pagan cuota alguna. La familia está afiliada al sistema de salud estatal.

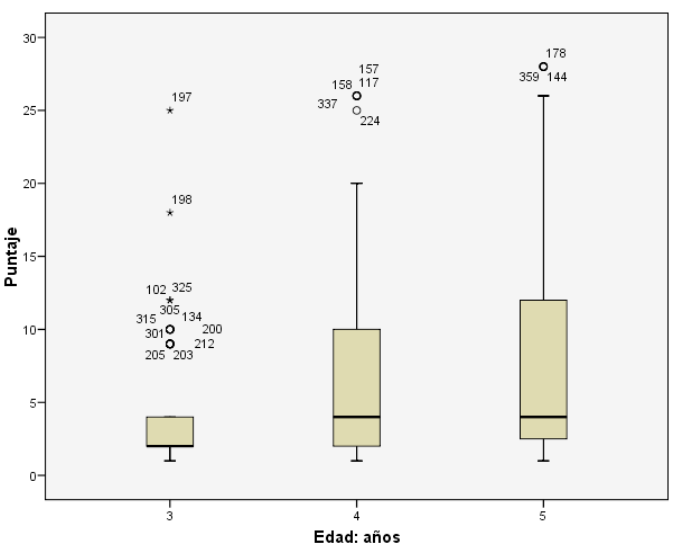

Figura 2. Distribución de los grupos de edad en función de los puntajes del proceso de solución.

Fuente: elaboración propia.

El $18 \%$ de las familias se sitúan en el nivel de menor pobreza; viven en una casa o apartamento propio, con acceso a todos los servicios públicos. La vivienda está construida en cemento y ladrillo, dispone de sala, comedor y piso de baldosa; dotación completa en baño(s) y cocina. Tanto el padre como la madre reciben ingresos de manera regular y los dos aportan para el sostenimiento de la familia. No tienen mayores dificultades para cubrir los gastos básicos, como arriendo, mercado, salud y ropa; los niños están vinculados al Hogar Comunitario y pagan una mensualidad mínima y las familias están afiliadas al sistema de salud.

La organización familiar muestra que el $57 \%$ de las familias cuenta con una red de apoyo para la crianza y cuidado de los niños: abuelos, tíos, primos, vecinos, que están vinculados con las actividades cotidianas de los niños. En las categorías convencionales que definen la organización familiar, $62 \%$ de las familias son nucleares, incluidas sus variaciones, extensa y compuesta; $9 \%$ de las familias son monoparentales sin red de apoyo. En estos casos 
las madres de familia asumen las responsabilidades económicas y de crianza4

Las prácticas de entretenimiento de las familias se manifiestan en los siguientes niveles: $69 \%$ reporta más de cuatro actividades de entretenimiento con los niños (Nivel 3); $23 \%$ y $8 \%$ quedan ubicadas en los niveles 1 y 2 , con 1 y 3 prácticas de entretenimiento, respectivamente. Un $55 \%$ de las familias se sitúa en los niveles medianamente alto (3) y alto (4) de prácticas de entretenimiento, $34 \%$ de las familias se ubican en el nivel medianamente bajo (2) y solamente el $11 \%$ están en el nivel bajo (1).

En cuanto a las prácticas de protección y cuidado, las familias tienden a ubicarse en el Nivel 2 y 3 (28 y $31 \%$, respectivamente). El $18 \%$ y el $23 \%$ se ubican en los niveles 1 y 4 , respectivamente. El $52 \%$ de las familias imponen entre 7 y 10 prohibiciones

4 Para la organización familiar no se identificaron niveles. Por esto no se incluye en el análisis de correspondencias. y actividades con límite (nivel 3: $41 \%$ y 4: $11 \%$ ); $30 \%$ alcanzan el nivel 2 y el $18 \%$, el nivel 1 . En el $72 \%$ de casos, la madre es quien impone y regula las normas. El $19 \%$ de las familias presenta el nivel más alto de expectativas (4) relativas a la escolaridad, el tipo de trabajo y las cualidades sociales que sus hijos deben alcanzar, además reportan acciones efectivas para el cumplimiento de las mismas. El $39 \%$ manejan expectativas medias altas y $21 \%$, medias bajas y bajas.

\section{Análisis de Correspondencias Múltiples}

Los resultados del análisis de correspondencia revelan que un puntaje alto en el desarrollo de la clasificación (CARA) se relaciona con nivel de pobreza bajo (NPB) y prácticas altas de regulación del comportamiento (PRCA), de protección y cuidado (PPCA), de formación y de entretenimiento. Un puntaje bajo en desarrollo de la clasificación

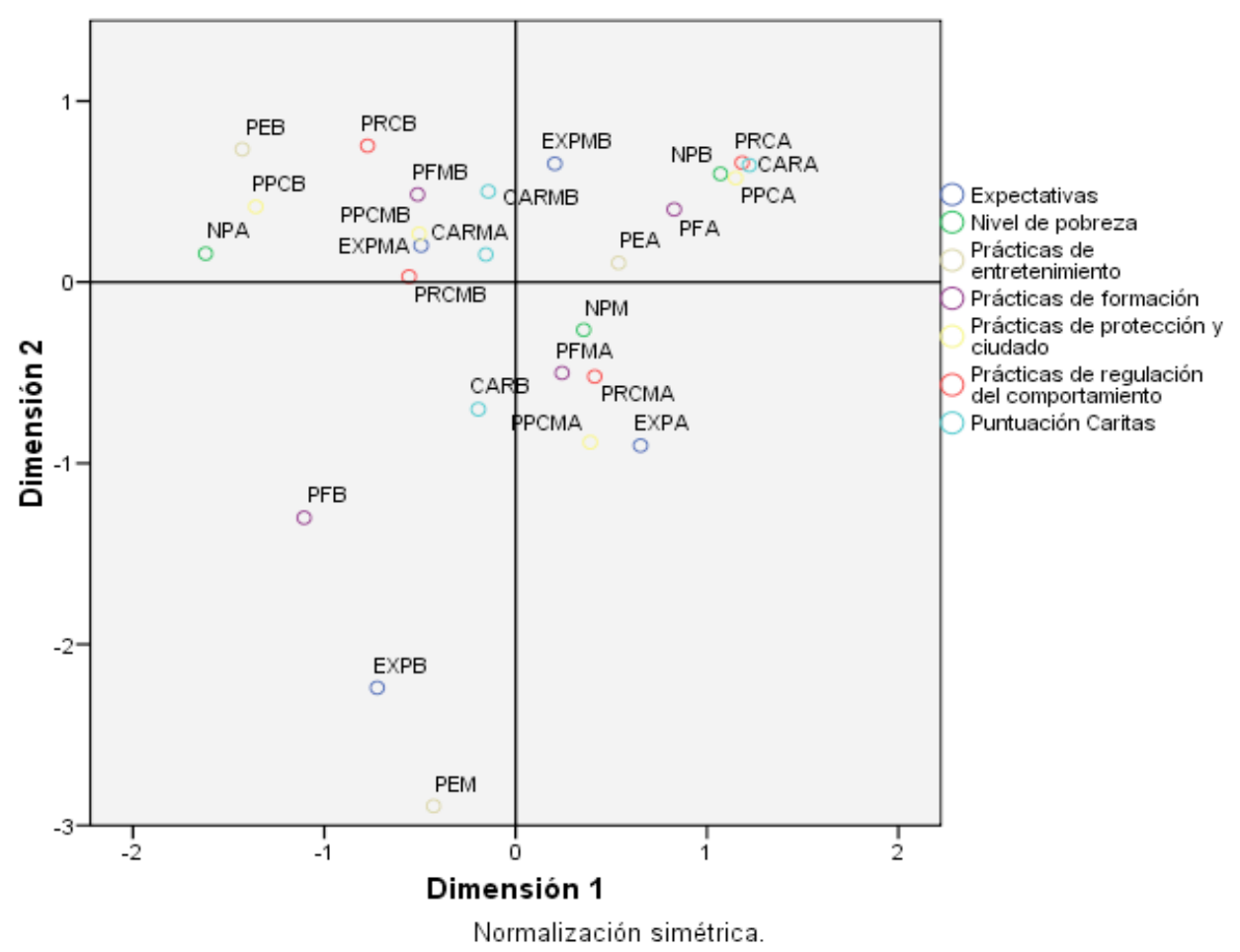

Figura 3. Relación entre desarrollo de la clasificación y dimensiones del contexto familiar. Fuente: elaboración propia. 
(CARB) se relaciona con prácticas de formación bajas (PFB) y expectativas bajas (EXPB).

\section{Análisis y discusión de los resultados}

El análisis y discusión de los resultados se centra en tres aspectos: una descripción del desarrollo cognitivo de los niños a partir del uso de la clasificación, la caracterización de los contextos de interacción familiar y las relaciones entre los dos componentes de esta investigación: desarrollo cognitivo y contextos.

\section{Desarrollo cognitivo}

Los resultados obtenidos muestran que el porcentaje de niños que logran un desempeño exitoso, se incrementa con la edad, asimismo, en el proceso de solución, aunque el porcentaje más alto de niños en todas las edades queda ubicado en los puntajes 2 y 4, el porcentaje de niños que alcanzan los niveles más altos tiende a aumentar en función de la edad. Los niños que se sitúan en un nivel bajo (puntajes 2 y 4) en el proceso de solución encuentran la carita, pero sus estrategias, y por tanto los esquemas de acción que la fundamentan, son menos complejos: en ambos casos utilizan deícticos siguiendo un orden, pero en el segundo, empiezan a formular alguna pregunta basada en relaciones de semejanza y diferencia, al establecer correspondencia entre cada lámina que señalan y la pregunta "ieste?" en un orden determinado, lo que les permite encontrar la carita, pero no se centran en las relaciones de semejanza y diferencia, base de la clasificación.

En cambio, los niños que obtienen un nivel de clasificación alto, no siempre identifican la cara que el investigador escoge, pero utilizan estrategias que los llevan a encadenar simultáneamente preguntas sobre los rasgos de los atributos. Para utilizar esta estrategia (8), los niños establecen: primero, diferencias entre los rasgos de un mismo atributo: género: niño o niña; semejanzas entre los elementos de una clase: si el entrevistador acepta que la carita escogida es la de una niña, entonces los niños deben continuar manejando la clase caritas de niñas; segundo, relación de pertenencia inclu- siva: la carita que busca pertenece a la clase de las caritas de niñas; tercero, intersección de una clase con otra clase: intersectan la clase de las caritas de niñas con la clase de las que tienen camisa roja, esto les exige crear una nueva clase con el color de la camisa; cuarto, intersecciones sucesivas entre más de dos clases en una operación multiplicativa compleja: la clase de las caritas de niñas y la clase de las que tienen la camisa roja se intersectan con las que sonríen y las que son negras ${ }^{5}$ y quinto, inclusión entre clases: la clase de las que tienen camisa roja y las que sonríen están incluidas en la clase de las niñas. En el caso de la estrategia 7, los niños deben aceptar que el rasgo escogido no corresponde con el de la carita que buscan; entonces, ante la respuesta negativa del(de la) entrevistador(a), deben escoger el rasgo complementario. Por ejemplo, si escogen la carita triste, la respuesta del entrevistador es: no; deben entonces continuar trabajando con el rasgo complementario: las no tristes (que son sonrientes, o con la boca abierta como dicen algunos niños). Estos resultados permiten señalar, que a pesar de la dificultad que el problema de clasificación presentó para los niños, a medida que la edad avanza, se presentan cambios que dan cuenta de su desarrollo cognitivo.

El uso de una situación de resolución de problemas y del análisis microgenético hizo posible identificar los esquemas de acción de los niños al resolver el problema y, a la vez, ofrecer una explicación de las diferencias que se generan al describir el desarrollo cognitivo desde la perspectiva del desempeño y de los procesos de solución. El problema de clasificación utilizado no es un test de desarrollo, sino una SRP que permitió describir las estrategias y los esquemas de acción que las fundamentan, esto no es posible con los tests convencionales centrados en el desempeño exitoso de los niños. Cualquier niño que utiliza la estrategia 2 puede llegar a identificar la carita escogida con un desempeño exitoso; pero las exigencias cognitivas de este tipo de estrategias son menores que las requeridas para una estrategia del nivel más alto. El análisis microgénetico de los

5 Descubra el lector cual es la carita escogida, según este ejemplo. 
esquemas de acción que subyacen a las estrategias, permitió inferir la actividad mental del niño desde la perspectiva de lo que saben y pueden hacer y no desde la perspectiva del observador externo que rastrea el buen desempeño.

En esta investigación se estudia el desarrollo cognitivo desde una perspectiva macro, es decir, en términos de cambios a través de períodos largos de tiempo, sin hacer seguimiento a un mismo individuo a lo largo de períodos cortos, utilizando diferentes tipos de tareas. Sin embargo, los resultados en la Figura 2 muestran una de las características del desarrollo: la variabilidad en el cambio y permiten señalar que niños de la misma edad utilizan todo tipo de estrategias para resolver el problema, dando cuenta de una variabilidad interindividual en su desarrollo cognitivo (Flynn \& Siegler, 2007; Miller \& Coyle, 1999). Igualmente, niños de todas las edades, incluido un niño de tres años, utilizan estrategias del más alto nivel. Para estudios posteriores, se recomienda la combinación de estudios de micro y macrodesarrollo que permitan caracterizar la variabilidad y la discontinuidad en las trayectorias del desarrollo (Puche, 2008).

\section{Contextos de interacción familiar}

Los contextos identificados se caracterizan de la siguiente manera: el $25 \%$ de las familias se encuentran en el nivel de mayor pobreza y guardan relación con prácticas de entretenimiento, protección y cuidado y regulación del comportamiento que se ubican en un nivel bajo. Las familias de nivel de pobreza medio $(57 \%)$ se relacionan con expectativas altas, prácticas de formación y regulación del comportamiento medias altas. Las familias con menor nivel de pobreza (18\%) están relacionadas con prácticas de regulación del comportamiento, protección y cuidado, formación y entretenimiento altas. Estas relaciones entre el nivel socioeconómico de las familias y sus prácticas y expectativas permiten definir los contextos de interacción en los cuales crecen y se desarrollan los niños de este estudio. En otras palabras, la configuración de nivel de pobreza, prácticas y expectativas define los contextos de interacción familiar.

\section{Relación entre desarrollo cognitivo y contextos}

En este estudio el nivel de pobreza de la familia o estatus socioeconómico, como dimensión aislada, no está relacionado con el nivel de desarrollo cognitivo de los niños. En el nivel de pobreza bajo, en el que se sitúan los padres menos pobres, se encuentra relación entre prácticas de regulación del comportamiento, de protección y cuidado, formación y entretenimiento altas con desarrollo alto de la clasificación. En tanto que el desarrollo bajo de la clasificación se relaciona con prácticas de formación y con un nivel bajo en las expectativas de los padres. Estos resultados coinciden con los hallazgos de Orozco et al. (2009), quienes encontraron una relación no significativa entre el nivel socioeconómico de las familias y los logros obtenidos ante una tarea de resolución de problemas, en niños que viven con sus familias en condiciones socioeconómicas de "alta pobreza", en este caso se detectaron niños con altos niveles de logro. Asimismo, estos autores encuentran que las expectativas altas de los padres es la variable que mejor se contrapone a los logros de los niños, al igual que la vinculación de las prácticas maternas con los niveles de expectativas de los padres y con el logro de los niños.

Weatherholt, Harris, Burns y Clement (2006) presentan una serie de investigaciones previas, cuyos resultados indican que niños pertenecientes a niveles socioeconómicos bajos muestran niveles de atención mucho más bajos que niños de sectores socioeconómicos medios y, al mismo tiempo, que los niños de familias de bajos ingresos presentan bajas habilidades para leer y escribir y para alcanzar logros académicos, así como puntajes bajos en los tests de inteligencia. Los resultados del presente estudio, por el contrario, permiten señalar que no es el nivel socioeconómico de las familias de los niños lo que de manera aislada incide en sus habilidades y logros. Son las configuraciones de niveles de pobreza, prácticas y expectativas, las que definen los contextos de interacción, que pueden incidir en su conjunto en el desarrollo cognitivo de los niños. La propuesta es, por tanto, que la pobreza extrema impide que los padres alcancen con sus hijos la variedad y riqueza 
de prácticas que los menos pobres asumen, lo que parece generar expectativas menos altas en relación con su futuro y niveles de desarrollo más bajos.

La noción de pobreza que actualmente se maneja está ligada con las posibilidades de consumo de las familias y en algunas latitudes esta noción no funciona. Esta posición crítica llevó a trabajar con la noción de contextos de interacción familiar y no con la noción de estatus socioeconómico como el factor que afecta y, en algunos casos, puede incidir en el desarrollo cognitivo de los niños. La noción de contextos de interacción permitió encontrar la riqueza de las prácticas y de las expectativas de los padres y, lo más importante, la incidencia de esas prácticas en el desarrollo de los niños. La ausencia de las interacciones que las prácticas y expectativas altas suscitan, es lo que podría considerarse como la verdadera miseria.

\section{Referencias}

Amar, J. (1994). Educación infantil y desarrollo social. Barranquilla: Ediciones Uninorte.

Bronfenbrenner, U. (1979). The ecology of human development: Experiments by nature and design. Cambridge, MA: Harvard University Press.

Bruner, J. (1975). Poverty and childhood. Oxford Review of Education, 1(1), 31-50.

Chavajay, P. (2006). How Mayan mothers with different amounts of schooling organize a problem-solving discussion with children. International Journal of Behavioral Development, 30, 371-382.

Correa-Chávez, M. \& Rogoff, B. (2009). Children's attention to interactions directed to others: Guatemalan Mayan and European-American patterns. Developmental Psychology, 45(3), 630-641.

Daza, G. (1999). Los vínculos de los que la familia es capaz. Nómadas, 11, 28-43.

Flynn, E. \& Siegler, R. (2007). Measuring change: Current trends and future directions in microgenetic research. Infant and Child Development, 16, 135-149.

García-Coll, C. (1990). Developmental outcome of minority infants: A process-oriented look into our beginnings. Child Development, 61(2), 270-289.
Gauvain, M. (1995). Thinking in niches: Sociocultural influences on cognitive development. Human Development, 38, 25-45.

Gónzalez, M. (1999). Cambio Social y dinámica familiar. Nómadas, 11, 54-62.

Greenfield, P. \& Lave, J. (1982). Cognitive aspects of informal education. En D. Wagner \& H. Stevenson (Eds.), Cultural perspectives on child development (pp. 181-207). San Francisco: Freeman.

Greenfield, P., Maynard, A. \& Childs, C. (2000). History, culture, learning, and development. CrossCultural Research: The Journal of Comparative Social Science, 34(4), 351-374.

Karmiloff-Smith, A. (1994). Más allá de la modularidad. Madrid: Alianza.

Korenman, S., Miller J. \& Sjaastad, J. (1994). Long-term poverty and child development in the United States. Manuscrito no publicado, University of Minnesota, Saint Paul, EE. UU.

LeVine, R. (1977). Cultura, conducta y personalidad. Madrid: Akal Editores.

Marquez dos Santos, L., Neves dos Santos, D. \& Lima, M. (2010). Socioeconomic status quality of domestic environment, and cognition in early childhood. En A. C. Bastos \& E. Prabinovich (Eds.), Living in poverty: Developmental poetics of cultural realities (pp. 269-286). Charlott, NC: Information Age Publishing.

McLoyd, V. (1990). The impact of economic hardship on black families and children. Child Development, 61(2), 311-346.

McLoyd, V. (1998). Socioeconomic disadvantage and child development. American Psychologist, 53, 185 204.

Miller, P. \& Coyle, T. (1999). Developmental change: Lessons from microgenesis. En E. K. Scholnick, K. Nelson, S. A. Gelman \& P. H. Miller (Eds.), Conceptual development: Piaget's legacy (pp. 209-239). Mahwah, NJ: Erlbaum.

Myers, R. (2003). Reflexiones sobre indicadores del desarrollo de niños menores de 6 años [Teleconferencia]. Disponible en www.redprimerainfancia.org

Orozco, M., Ochoa, S. \& Sánchez, H. (2001). Prácticas culturales y educación de la niñez. Bogotá: Fundación Antonio Restrepo Barco. 
Orozco, M., Perinat, A. \& Sánchez, H. (2009). Cognitive development and interaction context. En A. C. Bastos \& E. Prabinovich (Eds.), Living in poverty: Developmental poetics of cultural realities (pp. 287308). Charlott, NC: Information Age Publishing.

Panel Study of Income Dynamics. (1997). Child Development Supplement. Disponible en http://www. isr.umich.edu/src/child-development/home.html

Pascual-Leone, J. \& Johnson, J. (1991). The psychological unit and its role in task analysis: A reinterpretation of object permanence. En M. Chandler $\&$ M. Chapman (Eds.), Criteria for competence: Controversies in the conceptualization and assessment of children's abilities (pp. 151-187). Hillsdale, NJ: Earlbaum.

Pascual-Leone, J. \& Johnson, J. (2004). A dialectical constructivist view of developmental intelligence. En O. Wilhelm \& R. W. Engle (Eds.), Handbook of understanding and measuring intelligence (pp. 177201). Thousand Oaks: Sage.

Piaget, J. \& Inhelder, B. (1976). Génesis de las estructuras lógicas elementales: clasificaciones y seriaciones. Buenos Aires: Editorial Guadalupe.

Puche, R. (2000). Formación de herramientas científicas en el niño pequeño. Bogotá: Arango Editores.

Puche, R. (2003). El niño que piensa y vuelve a pensar. Cali: Artes Gráficas del Valle Editores.

Puche, R. (2008). Érase una vez el desarrollo. En F. Larreamendy-Joerns, R. Puche \& A. Restrepo (Eds.), Claves para pensar el cambio: ensayo sobre psicología del desarrollo (pp. 27-69). Bogotá: Uniandes/CESO.

Rodrigo, M. (1994). Etapas, contextos, dominios y teorías implícitas en el conocimiento social. En M. Rodrigo (Ed.), Contexto y desarrollo social (pp. 21-43). Madrid: Síntesis.
Rodríguez, C. (2006). Del ritmo al símbolo. Madrid: Horsori.

Rogoff, B. (1993). Aprendices del pensamiento. El desarrollo cognitivo en el contexto social. Barcelona: Piadós.

Rogoff, B., Mistry, J., Göncü, A. \& Mosier, C. (1993). Guided participation in cultural activity by toddlers and caregivers. Monographs of the Society to Research in Child Development, 58(8, Número de serie 236).

Rogoff, B., Moore, L., Najafi, B., Dexter, A., CorreaChávez, M. \& Solís, J. (2007). Children's development of cultural repertoires through participation in everyday routines and practices. En J. E. Grusec \& P. D. Hastings (Eds.), Handbook of socialization (pp. 490-515). New York: Guilford.

Ross, D. \& Roberts, S. (1999). Income and child wellbeing: A new perspective on the poverty debate. Ottawa: Canadian Council on Social Development.

Shonkoff, J. \& Phillips, D. (2000). From neurons to neighborhoods. The science of early childhood development. Washington, DC: National Academy Press.

Siegler, R. (2000). The rebirth of children's learning. Child Development, 71, 26-35.

Thornton, U. (1998). La resolución infantil de problemas. Madrid: Morata.

Tudge, J. (2009). Children`s development under conditions of poverty. En A. C. Bastos \& E. Prabinovich (Eds.), Living in poverty: Developmental poetics of cultural realities (pp. 287-308). Charlott, NC: Information Age Publishing.

Valsiner, J. (2007). Culture in minds and societies. New Delhi: Sage.

Weatherholt, T., Harris, R., Burns, B. \& Clement, C. (2006). Analysis of attention and analogical reasoning in children of poverty. Applied Developmental Psychology, 27, 125-135. 